\section{Phase Change Memories}

\section{Amit Chaudhry*}

Institute of Engineering and Technology, Panjab University, Chandigarh, India

Nearly six decades ago, S. Ovshinsky started research in amorphous materials and in 1968, he reported that certain glasses exhibited a reversible change in resistivity upon a change in phase from amorphous state to crystalline state and vice versa.

Phase change memories (PCM) are non-volatile memory devices that use reversible phase change in amorphous materials like Germanium, Antimony and Tellurium $\left(\mathrm{Ge}_{2} \mathrm{Sb}_{2} \mathrm{Te}_{5}\right)$ alloy to store information. The phase reversal results in the change in the electrical resistivity of the material used in the memory operation. These materials are broadly classified as chalcogenide materials. The phase change is induced by heating the material through a resistive element using a current pulse of a fixed duration during the two modes of operation. These are called as SET and RESET operations. During RESET operation, the current pulse of During RESET operation, a current pulse of high magnitude is applied to the materials so as to melt a critical volume of the material. The pulse produces a temperature more than the melting point of the material. The material melts and starts cooling down immediately when the voltage across the material is removed after a few nanoseconds. The molten volume of the material cools rapidly and the atoms fail to reorganize and loose crystalline behavior and an amorphous state of very high resistance is formed. On the other hand, during SET operation, a moderate voltage is applied across the material for a relatively longer duration than the RESET pulse. The temperature of the material rises above the crystallization temperature, but below the melting temperature. This mode results in the transformation of the amorphous state to the crystalline state. This state is also called low resistive state.
There are several tough challenges to actually implement the PCM. One of challenges is the complex phase change switching process. The complete understanding of the amorphous materials changing their phase to achieve the desired phase is still very low. So, the accurate modeling of the process and implementing as a PCM cell is a tough task. As such very few models have been developed in this field which describes the PCM behavior in a true sense. The second challenge is the very high programming current required to create amorphous state in the memory element. The drift of the resistance with time in amorphous state called as structural relaxation is another problem for the operation of the PCM. Thirdly, the issue in PCM technology is the material selection itself. The main material used in the GST. The other materials which can be used in PCM are GeSb, Ge-Te, GeSeTe, $\mathrm{AgSbSe}_{2}$, SbSe and Ag-In-Sb-Te. So, material selection based on the resulting performance achieved is a challenging task. The desired performance of the PCM is measured by various parameters. One of the parameter is the appropriate crystallization speed. It means the material should crystallize fast within a few tens of nanoseconds. To bring down the RESET current pulse magnitude, the material should have relatively high resistivity in the crystalline phase. The crystallization temperature of the material should be high in order to operate the material at high temperatures. The material should not react with the surrounding materials in the memory cell during its repeated operations. The material should be easily deposited using the existing CMOS-compatible deposition techniques.

There are several companies involved in the research and production of phase change memories. Some of them are Intel, Ovonyx and STMicroelectronics.
*Corresponding author: Amit Chaudhry, Institute of Engineering and Technology, Panjab University, Sector-25, Chandigarh, India, Tel. 91-172-2534995; E-mail: amit_chaudhry01@yahoo.com

Received December 19, 2012; Accepted December 21, 2012; Published December 24, 2012

Citation: Chaudhry A (2013) Phase Change Memories. J Electr Electron Syst 2: e110. doi:10.4172/2332-0796.1000e110

Copyright: (c) 2013 Chaudhry A. This is an open-access article distributed unde the terms of the Creative Commons Attribution License, which permits unrestricted use, distribution, and reproduction in any medium, provided the original author and source are credited. 